\title{
Neuropsychiatric symptoms in Tanzanian HIV-infected children receiving long-term efavirenz treatment: a multicentre, cross-sectional, observational study
}

Lisa Van de Wijer, Deborah N. Mchaile, Quirijn de Mast, Blandina T. Mmbaga, Nanda N.J. Rommelse, Ashanti Duinmaijer, André J.A.M. van der Ven, Arnt F.A. Schellekens, Grace D. Kinabo

Lisa Van de Wijer and Deborah N. Mchaile contributed equally to the work. Arnt F.A. Schellekens and Grace D. Kinabo contributed equally to the work.

Department of Internal Medicine, Radboud university medical center, Nijmegen, The Netherlands (L Van de Wijer MSc, Q de Mast PhD, A Duinmaijer MSc, Prof A van der Ven PhD)

Department of Paediatrics, Kilimanjaro Christian Medical Centre, Moshi, Tanzania (D N Mchaile MMed, A Duinmaijer MSc, G D Kinabo PhD)

Kilimanjaro Clinical Research Institute, Moshi, Tanzania (B T Mmbaga PhD)

Karakter Child and Adolescent Psychiatry University Centre, The Netherlands (N N Rommelse PhD)

Department of Psychiatry, Donders Institute for Brain, Cognition and Behaviour, Radboud university medical center, Nijmegen, The Netherlands (N N Rommelse PhD, A F Schellekens PhD)

Nijmegen Institute for Scientist Practitioners in Addiction, Nijmegen, The Netherlands (A F Schellekens $\mathrm{PhD})$

Corresponding author:

Lisa Van de Wijer

Department of Internal Medicine, Radboud university medical center, PO Box 9101, 6500 HB Nijmegen, The Netherlands

E-mail: L.vandewijer@radboudumc.nl

Tel: +31243619610 Fax: +31243566336

Lancet HIV 2019; published online Feb 12. http://dx.doi.org/10.1016/S2352-3018(18)30329-1. 


\section{Summary}

Background Efavirenz is commonly prescribed for children with HIV infection, yet little is known about risks of neuropsychiatric side-effects. We aimed to compare competence (social involvement, activities, and school performance) and psychopathology (internalising and externalising problems), cognitive performance (intelligence and working memory), and adherence in Tanzanian children on an efavirenz-based versus a nonefavirenz-based regimen.

Methods In this multicentre, cross-sectional, observational study, we included consecutive children (aged 6-12 years) with HIV infection, on combination antiretroviral therapy (cART) for at least 6 months, and with viral loads of less than 1000 copies per mL from HIV care clinics of three primary health facilities and three referral hospitals in Moshi, Kilimanjaro, Tanzania. Children with acute illnesses, medication switch in the 6 months before the study visit, and any history of brain injury or developmental delay before cART initiation were excluded. All interviews and assessments were done by trained local research nurses under the supervision of a medical doctor. The primary outcomes, competence and psychopathology, were measured with the Child Behavior Checklist. We used ANCOVA to assess differences between groups. This study is registered with ClinicalTrials.gov, number NCT03227653.

Findings Between June 19, 2017, and Dec 14, 2017, 141 children were analysed, of whom $72(51 \%)$ used efavirenz-based cART and 69 (49\%) used non-efavirenz-based cART. After controlling for age, sex, and clinical and demographic confounders, we observed lower competence (adjusted mean difference $-2 \cdot 43$ [95\% $\mathrm{CI}-4 \cdot 19$ to $-0 \cdot 67], \mathrm{p}=0 \cdot 0071$ ), largely driven by lower school performance scores (adjusted mean difference $-0.91[-1.42$ to -0.40$], \mathrm{p}=0.00055)$, in the efavirenz group than in the non-efavirenz group. More total (adjusted mean difference 5.96 [95\% CI $-1 \cdot 12$ to $13 \cdot 04], \mathrm{p}=0 \cdot 098$ ) and internalising (adjusted mean difference $2 \cdot 00$ [-0.29 to $4 \cdot 29], \mathrm{p}=0 \cdot 086$ ) behavioural problems were seen in the efavirenz group than in the non-efavirenz group, although these findings were non-significant. No differences were found in externalising problems (adjusted mean difference $0 \cdot 78[95 \% \mathrm{CI}-1 \cdot 55$ to $3 \cdot 11], \mathrm{p}=0 \cdot 51$ ).

Interpretation Our results suggest that treatment with efavirenz in children is associated with a mild increase in neuropsychiatric symptoms, especially in children who receive doses higher than or equal to the WHO recommended doses for efavirenz. Clinical awareness and adequate follow-up of neuropsychiatric symptoms in efavirenz in children remain warranted.

Funding: Aidsfonds, Radboud university medical center.

Keywords: efavirenz, ART therapy, child, adverse effects, Africa South of the Sahara, central nervous system, neuropsychological tests, child behaviour, cognition, treatment adherence and compliance 


\section{Introduction}

In 2017, an estimated 1.8 million children worldwide were living with HIV, of whom 52\% received combination antiretroviral therapy (cART). ${ }^{1}$ The Tanzanian national HIV guidelines recommend including efavirenz in firstline cART for children aged 3 years or older., ${ }^{2,3}$ In children, efavirenz has several advantages compared with its alternatives nevirapine and ritonavir-boosted lopinavir, including superior antiviral efficacy, simplified cotreatment for tuberculosis, and lower risks for long-term metabolic and bone complications. ${ }^{4}$ Among adults on efavirenz, a third reports neuropsychiatric side-effects such as nightmares, depression, and hallucinations. ${ }^{5}$ Although most of these symptoms decrease after weeks to months, some can persist for years, with negative consequences for quality of life, adherence, and treatment discontinuation. ${ }^{6,7}$ Apart from neuropsychiatric symptoms, efavirenz has been linked to impaired neurocognitive performance. ${ }^{8}$ In part because of these tolerability concerns, the latest WHO guidelines ${ }^{9}$ no longer recommend efavirenz as the general drug of choice for people with HIV infection.

Although children generally seem to tolerate efavirenz well, data about neuropsychiatric side-effects remain inconclusive. ${ }^{10,11}$ Until now, only one clinical trial had published psychiatric data about children on efavirenz. By use of a brief behavioural screener, the Strengths and Difficulties Questionnaire (SDQ), the authors observed no differences in psychopathology between efavirenz and ritonavir-boosted lopinavir. ${ }^{12,}{ }^{13}$ By contrast, other studies $^{14,15}$ reported high frequencies (36-39\%) of neuropsychiatric symptoms. Several case reports ${ }^{14,16,17}$ also described the occurrence of severe symptoms, including psychosis, ataxia, and seizures. These discrepancies between studies might be partly explained by the large heterogeneity between cohorts, small sample sizes, or lack of validated tools for comprehensive paediatric psychiatric assessments. ${ }^{11}$ Potential long-term neuropsychiatric side-effects need to be identified because these symptoms might interfere with neurodevelopment and psychosocial maturation. In our study, we aimed to comprehensively assess behaviour and cognition in children with HIV infection in Tanzania by use of validated psychiatric tools. Our primary goal was to compare competence and psychopathology between children treated with efavirenz-based cART and children treated with alternative, non-efavirenz-based cART (nevirapine or ritonavir-boosted lopinavir). Second, we aimed to compare cognitive performance and adherence between treatment groups. We hypothesised that treatment with efavirenz would be associated with lower competence, more psychopathology, reduced cognitive performance, and lower adherence compared with alternative cART. 


\section{Methods}

\section{$\underline{\text { Study design and participants }}$}

In this multicentre, cross-sectional, observational study, we enrolled consecutive HIV-1-positive children who were registered at the HIV clinics at Kilimanjaro Christian Medical Centre (a tertiary referral hospital), Mawenzi Regional Hospital (a referral hospital), Kibosho Hospital, Majengo Health Centre, Pasua Health Centre, and Kiboriloni Dispensary in Moshi District, Kilimanjaro Region, Tanzania. Inclusion criteria were age 6-12 years, presence of a caretaker (parent or guardian who was part of the upbringing of the child), treatment with cART for at least 6 months, and an HIV-1 RNA count of less than 1000 copies per mL within 12 months of the study visit. Exclusion criteria were acute illnesses, medication switch in the 6 months before the study visit, and any history of brain injury or developmental delay before cART initiation.

The institutional review boards of the Kilimanjaro Christian Medical University College (Moshi, Kilimanjaro, Tanzania; number 2028) and the National Institute for Medical Research (Dar es Salaam, Tanzania; number 2447) approved the study protocol. Written informed consent in Kiswahili from parents or guardians of all participants was obtained before enrolment. Children aged 12 years provided oral assent.

\section{Procedures}

An overview of the study methods and procedures is provided in the appendix (p 20). Caretakers completed an extensive questionnaire on the demographic and socioeconomic status of the household, HIV disclosure, and the health status of the child. Clinical data, including HIV-1 RNA results, were obtained retrospectively from the child's clinical file. We assessed nutrition using height and body-mass index for age-adjusted Z scores (2007 WHO growth reference data for children and adolescents aged 5-19 years).

For behavioural assessments, we used the Child Behavior Checklist for ages 6-18 years (CBCL/6-18). The CBCL is a standardised 120-item questionnaire that provides a comprehensive caretaker report of a child's competence, and behavioural and emotional problems. ${ }^{18}$ The competence section of the CBCL includes questions on the child's activities (sports, hobbies, and jobs), social involvement (organisations and friends), and school performance (grades and special classes). The problem section consists of syndrome scores that together yield an internalising (anxiety or depression, withdrawn behaviour, or somatic complaints) and externalising (rule-breaking or aggressive behaviour) problem score. The total problem score is the sum of all problem items. Four main scores can be calculated: total competence, total problems, internalising problems, and externalising problems. Raw scores were converted into age-specific, sex-specific, and (Kenyan) population-specific T scores to calculate the number of children within the borderline clinical range. ${ }^{18}$ The CBCL is available in Kiswahili and has been previously applied in HIV-positive children in sub-Saharan Africa. ${ }^{19}$

We applied Raven's Coloured Progressive Matrices (RCPM) and the digit span test to assess cognitive performance. RCPM was used to measure general non-verbal cognitive ability. RCPM consists of 36 multiplechoice coloured matrices in which items are ordered by increasing difficulty. RCPM is non-verbal, easy, and rapid to administer and score, and has been validated for use in children living in Kenya. ${ }^{20}$

The digit span test (from the Wechsler Intelligence Scale for Children-III) was used to measure short-term and working memory. ${ }^{21}$ 
A string of numbers was read out to the child, who was asked to repeat them in the same order (digit forwards) or reversed order (digit backwards). The test was discontinued if the child failed on two consecutive trials. The total score corresponds to the maximum number of digits the child repeated correctly. The digit span test has been used in HIV-positive children in sub-Saharan Africa. ${ }^{22}$

For adherence, we used a questionnaire designed by the Pediatric European Network for Treatment of AIDS. ${ }^{23}$ To determine non-adherence, caretakers were asked how many times the child had missed his or her medication in the previous 3 days. Next, caretakers marked adherence since the last clinical visit on a visual analogue scale. In addition, structured questions were asked about other aspects of cART adherence. Non-adherence was defined as one or more missed doses over the previous 3 days or marking less than $100 \%$ adherence since the last clinical visit on the visual analogue scale.

Finally, we applied a CNS symptom checklist with symptoms that have been linked to efavirenz in the scientific literature. ${ }^{7,11}$ Caretakers and children were asked whether the child had experienced any of these symptoms in the week before the study visit.Interviews and cognitive assessments were done by six local research nurses, who were experienced in paediatric HIV treatment and counselling. During a 1-week programme, a Dutch medical doctor (LVdW) and a Tanzanian paediatrician (DNM) trained the research nurses in conducting the interview and cognitive assessments. The research nurses were supervised by LVdW or DNM throughout the study period.

Before enrolment, children were screened by their own physician or the study doctor for acute illnesses and signs of gross developmental delay. The study visit consisted of two parts. To minimise information bias, caretakers were informed about the study background (CNS side-effects of HIV drugs) and procedures, without disclosing our hypothesis. After informed consent, a research nurse interviewed the caretaker and child on adherence and general wellbeing. Next, a second research nurse interviewed the caretaker about demographic and clinical parameters, and read out the items of the CBCL, while the initial nurse continued with cognitive assessment of the child. To optimise cognitive test performance, all visits took place in quiet, private rooms and were completed before 1300 h. Data were stored securely and anonymously in the Castor Electronic Data Capture programme (Amsterdam, Netherlands).

\section{$\underline{\text { Outcomes }}$}

Primary outcome measures were competence and psychopathology (internalising and externalising symptoms), as defined by the CBCL. Secondary outcome measures were non-verbal cognitive ability, short-term and working memory, and non-adherence. Finally, we added CNS symptoms linked to efavirenz in the scientific literature as exploratory outcomes. The characteristics of the outcome variables of this study are presented in the appendix (p 13).

\section{$\underline{\text { Data analysis }}$}

The study required 128 participants (64 participants per group) to detect a medium effect difference in CBCL total problem scores (Cohen's $f=0 \cdot 25$, equal to a difference of $3 \cdot 9$ in CBCL total problem T scores), ${ }^{19}$ with $80 \%$ power and $\alpha$ set at $0 \cdot 05$. We compared general characteristics between the efavirenz group and non-efavirenz group using Student's $t$ test (or Mann-Whitney $U$ test) for continuous variables and Pearson's $\chi^{2}$ test (or Fisher's 
exact test) for categorical variables. We used ANCOVA to assess between-group differences in the raw main scores (total competence, total problems, internalising problems, and externalising problems). Significant differences were assessed by post-hoc ANCOVA for the individual competence or syndrome scores (eg, anxiety or depression for internalising problems). We assessed between-group differences in cognitive performance scores with ANCOVA and in non-adherence with Pearson's $\chi^{2}$ test. We analysed the presence of CNS symptoms using logistic regression models. The following covariates were included because of group differences or their clinical relevance in disease severity and mental health: age, sex, parental loss, HIV disclosure, previous hospital admission, history of tuberculosis treatment, previous treatment failure, and cART duration. During data collection, we discovered that 28 (39\%) of 71 children received lower efavirenz doses than recommended by WHO. ${ }^{3}$ Thereafter, we decided to add a subgroup analysis in which children who were prescribed lower efavirenz doses than recommended by WHO were excluded. We analysed data using SPSS Statistics, version 25.

This study is registered with ClinicalTrials.gov, number NCT03227653.

\section{Role of the funding source}

The funders of the study had no role in study design, data collection, data analysis, data interpretation, or writing of the report. The corresponding author had full access to all the data in the study and had final responsibility for the decision to submit for publication. 


\section{Results}

Between June 19, 2017, and Dec 14, 2017, 144 children were enrolled at the six study sites. Three children were excluded from analysis because of age less than 6 years, unreported severe developmental delay, and cART switch the week before the study visit (figure). 141 children were analysed, of whom 72 (51\%) used efavirenzbased cART and 69 (49\%) used non-efavirenz-based cART. Groups were balanced for age, sex, and nutritional status parameters (table 1). Socioeconomic characteristics did not differ between groups (appendix p 6). Children in the non-efavirenz group used cART for a longer time ( $\mathrm{p}<0 \cdot 0001)$, had a more advanced WHO clinical stage at start of $\mathrm{cART}(\mathrm{p}=0.011)$, had more often experienced previous treatment failure $(\mathrm{p}<0 \cdot 0001)$, and had more often been admitted to the hospital $(\mathrm{p}=0 \cdot 0016)$ than children in the efavirenz group. Children in the efavirenz group more often had received tuberculosis treatment $(\mathrm{p}=0.022)$ and had more often had their own HIV status disclosed $(\mathrm{p}=0 \cdot 016)$ than children in the non-efavirenz group. In addition, we observed a non-significant higher prevalence of parental loss in the efavirenz group than in the non-efavirenz group $(\mathrm{p}=0 \cdot 078)$. With the exception of co-trimoxazole (table 1), no relevant co-medication was used. At the time of analysis, none of the children was undergoing tuberculosis treatment. Ten (14\%) of 69 children in the non-efavirenz group had previously discontinued efavirenz. Efavirenz had been switched to nevirapine in three (30\%) of ten children or to ritonavirboosted lopinavir in seven (70\%) of ten children after a median of 407 days (IQR 179-975) for the following reasons: treatment failure (seven [70\%] of ten children), neuropsychiatric side-effects (one [10\%] of ten children), end of tuberculosis treatment (one [10\%] of ten children), and unspecified (one [10\%] of ten children). 


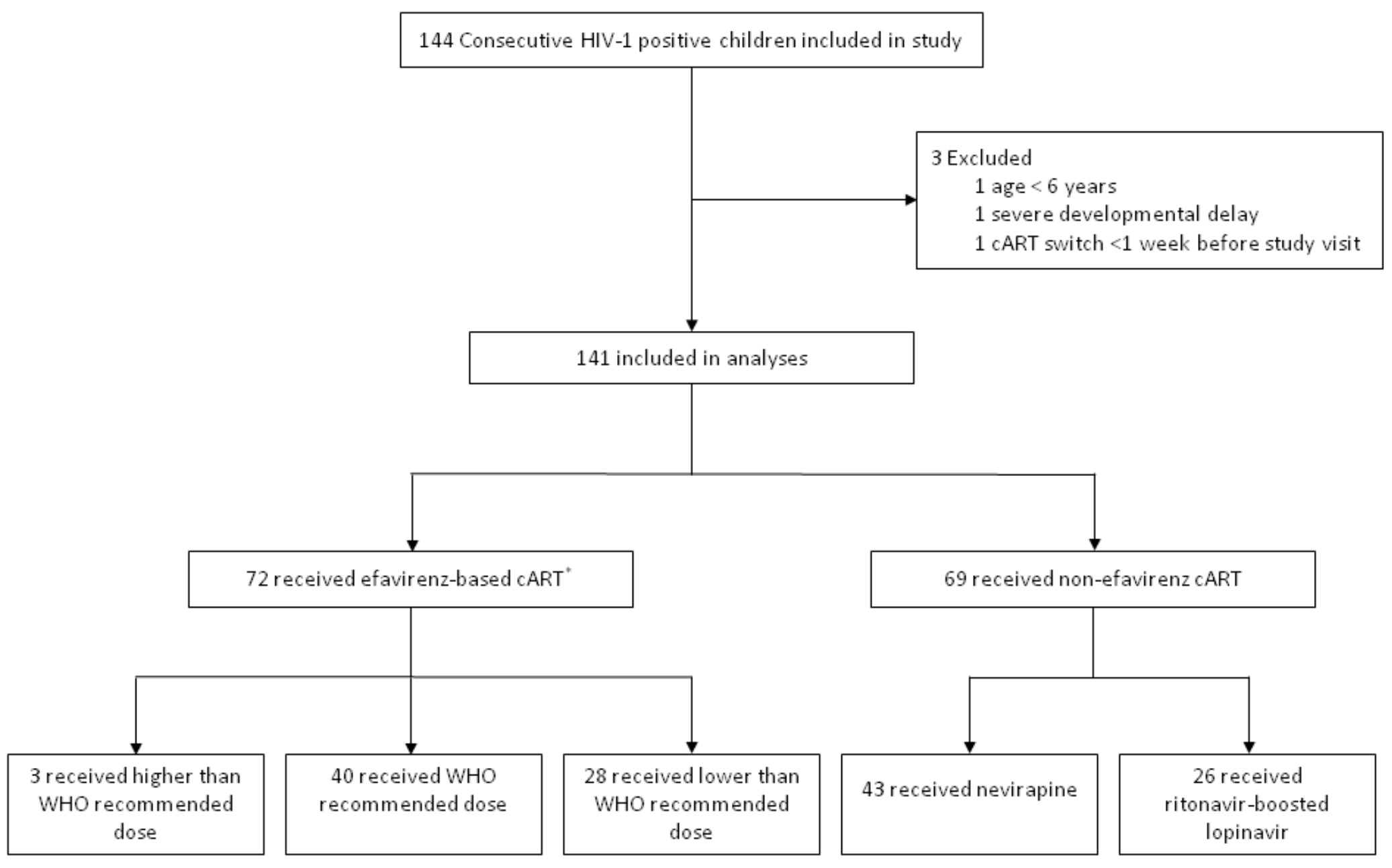

Figure Study overview

cART=combination antiretroviral therapy. *The efavirenz dose $(\mathrm{mg} / \mathrm{kg})$ could not be determined for one of the participants because of missing bodyweight data. 
Table 1. Clinical and demographic characteristics

\begin{tabular}{|c|c|c|c|}
\hline Characteristic & $\begin{array}{c}\text { Non-efavirenz } \\
(n=69)\end{array}$ & $\begin{array}{c}\text { Efavirenz } \\
(\mathrm{n}=72)\end{array}$ & P-value \\
\hline Age, years & $9 \cdot 4(1 \cdot 8)$ & $9.9(1.9)$ & $0 \cdot 094$ \\
\hline Sex & & & $0 \cdot 61$ \\
\hline Male & $33(48 \%)$ & $31(43 \%)$ &.$\cdot$ \\
\hline Female & $36(52 \%)$ & $41(57 \%)$ & .. \\
\hline WHO BMI z-score ${ }^{*}$ & $-0 \cdot 80(1 \cdot 09)$ & $-0 \cdot 84(1 \cdot 03)$ & $0 \cdot 81$ \\
\hline WHO height z-score ${ }^{*}$ & $-1 \cdot 00(0 \cdot 80)$ & $-1 \cdot 16(1 \cdot 04)$ & $0 \cdot 33$ \\
\hline HIV-RNA $<40$ copies per $\mathrm{mL}$ & $54(78 \%)$ & $54(75 \%)$ & 0.69 \\
\hline Time since last HIV-RNA measurement, median (IQR) days & $100(56-168)$ & $133(57-198)$ & $0 \cdot 074$ \\
\hline Vertical transmission & $69(100 \%)$ & $72(100 \%)$ &.. \\
\hline \multicolumn{4}{|l|}{ WHO clinical stage at start cART } \\
\hline Stage 1 & $10 / 68(15 \%)$ & $9 / 70(13 \%)$ & $0 \cdot 011$ \\
\hline Stage 2 & $16 / 68(24 \%)$ & $29 / 70(41 \%)$ & \\
\hline Stage 3 & $21 / 68(31 \%)$ & $25 / 70(36 \%)$ & \\
\hline Stage 4 & $21 / 68(31 \%)$ & $7 / 70(10 \%)$ & \\
\hline Prior treatment failure $^{\dagger}$ & $23(33 \%)$ & $1(1 \%)$ & $<0 \cdot 0001$ \\
\hline Time on cART, median (IQR) years & $7 \cdot 6(5 \cdot 4-8 \cdot 7)$ & $3 \cdot 5(2 \cdot 1-5 \cdot 6)$ & $<0 \cdot 0001$ \\
\hline Time on efavirenz, median (IQR) years & .. & $3 \cdot 1(2 \cdot 0-5 \cdot 1)$ & .. \\
\hline Prior efavirenz discontinuation & $10(14 \%)$ &.. & .. \\
\hline \multicolumn{4}{|l|}{ cART backbone } \\
\hline abacavir + lamivudine & $23(33 \%)$ & $17(24 \%)$ & $0 \cdot 11$ \\
\hline zidovudine + lamivudine & $45(65 \%)$ & $52(72 \%)$ & \\
\hline tenofovir disproxil fumarate + lamivudine & $0(0)$ & $3(4 \%)$ & \\
\hline zidovudine + abacavir + lamivudine & $1(1 \%)$ & $0(0)$ & \\
\hline \multicolumn{4}{|l|}{ cART $3^{\text {rd }}$ drug } \\
\hline nevirapine & $43(62 \%)$ & .. & .. \\
\hline lopinavir-ritonavir & $26(37 \%)$ & .. & \\
\hline History of tuberculosis treatment & $12(17 \%)$ & $25(35 \%)$ & $0 \cdot 022$ \\
\hline Co-trimoxazol treatment & $33(48 \%)$ & $36(50 \%)$ & 0.95 \\
\hline Previous hospital admission ${ }^{*}$ & $53(77 \%)$ & $36(50 \%)$ & $0 \cdot 0016$ \\
\hline \multicolumn{4}{|l|}{ Caretaker's relationship } \\
\hline Parent & $49(71 \%)$ & $41(57 \%)$ & $0 \cdot 36$ \\
\hline Relative & $16(23 \%)$ & $25(35 \%)$ & \\
\hline Orphanage caretaker & $2(3 \%)$ & $2(3 \%)$ & \\
\hline Other & $2(3 \%)$ & $4(6 \%)$ & \\
\hline \multicolumn{4}{|l|}{ Parental loss } \\
\hline None (both alive) & $44(64 \%)$ & $33(46 \%)$ & $0 \cdot 078$ \\
\hline Single & $19(28 \%)$ & $26(36 \%)$ & \\
\hline Double & $6(9 \%)$ & $13(18 \%)$ & \\
\hline \multicolumn{4}{|l|}{ School attendance } \\
\hline Nursery school & $8(12 \%)$ & $10(14 \%)$ & $0 \cdot 50$ \\
\hline Primary school & $61(88 \%)$ & $60(83 \%)$ & \\
\hline No school & $0(0)$ & $2(3 \%)$ & \\
\hline HIV-status disclosed & $13(19 \%)$ & $27(38 \%)$ & $0 \cdot 016$ \\
\hline
\end{tabular}

Data are mean (SD), $n(\%)$, median (IQR), or $n / N(\%)$. Data were analysed with Student's $t$ test (or Mann-

Whitney $U$ test) for continuous variables and Pearson's $\chi^{2}$ test (or Fisher's exact) for categorical variables. $c A R T=$ combination antiretroviral therapy.

* Z scores calculated using WHO 2007 growth reference data for children and adolescents aged 5-19 years. + Defined as any immunological, virological, or clinical failure documented in the medical file.

$\neq$ Defined as any previous hospital admission reported by the caretaker or documented in the medical file. 
Overall, 15 (22\%) of 69 children in the non-efavirenz group and 21 (30\%) of 71 children in the efavirenz group scored below the normal range for total competence (defined as scores below the 16th age-specific, sex-specific, and Kenyan population-specific percentile; appendix p 7). Table 2 shows the mean (SD) raw scores on the CBCL per treatment group, as well as the unadjusted and the confounder-adjusted models. In general, the covariates age and duration of cART had the largest effect on the confounder-adjusted models (appendix p 17). Children in the efavirenz group scored lower than children in the non-efavirenz group on the competence scale (adjusted mean difference $-2 \cdot 43$ [95\% CI $-4 \cdot 19$ to $-0 \cdot 67], \mathrm{p}=0 \cdot 0071$ ). Post-hoc analyses showed that these lower competence scores were primarily driven by lower school performance scores (adjusted mean difference $-0.91[95 \% \mathrm{CI}-1 \cdot 42$ to $-0 \cdot 40], \mathrm{p}=0 \cdot 00055)$.

Except for internalising problems, psychopathology was relatively uncommon in our study population. Scores above the normal range (defined as scores above the 84th age-specific, sex-specific, and Kenyan populationspecific percentile; appendix p 7) were seen in eight (12\%) of 69 children in the non-efavirenz group versus nine (13\%) of 72 children in the efavirenz group for total problems, in 17 (25\%) of 69 children versus $22(31 \%)$ of 72 children for internalising problems, and in six (9\%) of 69 children versus seven $(10 \%)$ of 72 children for externalising problems. We observed non-significantly higher total problem scores (adjusted mean difference $5 \cdot 96$ [95\% CI $-1 \cdot 12$ to $13 \cdot 04], \mathrm{p}=0 \cdot 098$ ) and higher internalising problem scores (adjusted mean difference $2 \cdot 00$ $[-0 \cdot 29$ to $4 \cdot 29], \mathrm{p}=0 \cdot 086)$ in the efavirenz group than in the non-efavirenz group, but we found no differences in externalising problems (adjusted mean difference $0 \cdot 78[-1 \cdot 55$ to $3 \cdot 11], \mathrm{p}=0 \cdot 51$ ).

Using South African RCPM norm scores for children, which measure general non-verbal cognitive ability, four $(6 \%)$ of 69 children in the non-efavirenz group and two (3\%) of 72 children in the efavirenz group scored below the 10th percentile (appendix p 8), indicating that non-verbal cognitive ability was largely unaffected in both groups. We found no group differences in general non-verbal cognitive ability, and we did not observe an effect of efavirenz on short-term memory or working memory (table 2). Non-adherence was reported by 24 (35\%) of 69 children in the non-efavirenz group and $20(28 \%)$ of 72 children in the efavirenz group $(\mathrm{p}=0 \cdot 47$; appendix $\mathrm{p}$ 9). Additional information on adherence is summarised in the appendix ( $\mathrm{p} 9$ ).

Finally, we explored the presence of CNS symptoms that have been linked to efavirenz in the scientific literature (table 3; appendix p 18). In accordance with the results from the CBCL, children in the efavirenz group more frequently reported problems paying attention at school (19 [26\%] of 72 children $v s$ eight [12\%] of 69 children), depressed or anxious feelings (17 [24\%] of 72 children vs six [9\%] of 69 children), and feelings of uneasiness (20 [28\%] of 72 children vs 12 [17\%] of 69 children) than did children in the non-efavirenz group (table 3 ). Abnormal dreaming was less frequently reported among children in the efavirenz group than among children in the non-efavirenz group (table 3). 
Table 2. Behavioural and neurocognitive test scores

\begin{tabular}{|c|c|c|c|c|c|c|}
\hline \multirow{2}{*}{ Item } & \multirow{2}{*}{ Non-efavirenz $(n=69)$} & \multirow{2}{*}{ Efavirenz $(n=72)$} & \multirow{2}{*}{$\begin{array}{c}\text { Crude model } \\
\text { Mean difference }(95 \% \mathrm{CI})\end{array}$} & \multicolumn{3}{|c|}{ Adjusted model } \\
\hline & & & & P-value & Mean difference (95\% CI) & P-value \\
\hline \multicolumn{7}{|c|}{ Child Behaviour Checklist competence scores" } \\
\hline Total Competence & $23 \cdot 6(4 \cdot 1)$ & $23 \cdot 4(4 \cdot 1)$ & $-0 \cdot 12(-1.49$ to $1 \cdot 25)$ & $0 \cdot 86$ & $-2 \cdot 43(-4 \cdot 19$ to $-0 \cdot 67)$ & $0 \cdot 0071$ \\
\hline Activities & $10 \cdot 4(2 \cdot 7)$ & $10 \cdot 4(2 \cdot 2)$ & $0.08(-0.74$ to 0.91$)$ & $0 \cdot 84$ & $-0.90(-2.01$ to 0.21$)$ & $0 \cdot 11$ \\
\hline Social & $8.5(1.8)$ & $8.9(2 \cdot 0)$ & $0 \cdot 38(-0.26$ to $1 \cdot 02)$ & $0 \cdot 24$ & $-0.61(-1.46$ to 0.23$)$ & $0 \cdot 15$ \\
\hline School & $4 \cdot 7(0 \cdot 9)$ & $4 \cdot 1(1 \cdot 3)$ & $-0.59(-0.97$ to $-0 \cdot 22)$ & $0 \cdot 0022$ & $-0.91(-1.42$ to -0.40$)$ & $0 \cdot 00055$ \\
\hline \multicolumn{7}{|l|}{ Child Behaviour Checklist problem score } \\
\hline Total problems & $25 \cdot 3(13 \cdot 0)$ & $27 \cdot 4(17 \cdot 6)$ & $2 \cdot 07(-3 \cdot 11$ to $7 \cdot 25)$ & $0 \cdot 43$ & $5 \cdot 96(-1 \cdot 12$ to $13 \cdot 04)$ & $0 \cdot 098$ \\
\hline Internalizing problems & $8 \cdot 0(3 \cdot 9)$ & $9 \cdot 6(5 \cdot 8)$ & $1.60(-0.06$ to 3.25$)$ & 0.058 & $2 \cdot 00(-0.29$ to 4.29$)$ & $0 \cdot 086$ \\
\hline Externalizing problems & $5 \cdot 8(4 \cdot 7)$ & $5 \cdot 7(5 \cdot 5)$ & $-0 \cdot 15(-1.85$ to 1.56$)$ & $0 \cdot 87$ & $0.78(-1.55$ to $3 \cdot 11)$ & 0.51 \\
\hline \multicolumn{7}{|l|}{ Other problems } \\
\hline Social problems & $3 \cdot 0(2 \cdot 4)$ & $3 \cdot 1(2 \cdot 8)$ & $0.08(-0.79$ to 0.96$)$ & $0 \cdot 85$ & $0.61(-0.61$ to $1 \cdot 83)$ & $0 \cdot 32$ \\
\hline Thought problems & $1 \cdot 5(1 \cdot 9)$ & $1 \cdot 7(2 \cdot 3)$ & $0.19(-0.51$ to 0.88$)$ & $0 \cdot 60$ & $0.81(-0.16$ to $1 \cdot 78)$ & $0 \cdot 10$ \\
\hline Attention problems & $3 \cdot 4(2 \cdot 7)$ & $3 \cdot 5(3 \cdot 5)$ & $0 \cdot 11(-0.93$ to $1 \cdot 14)$ & $0 \cdot 84$ & $1 \cdot 19(-0.21$ to $2 \cdot 59)$ & $0 \cdot 096$ \\
\hline \multicolumn{7}{|l|}{ Neurocognitive scores } \\
\hline Raven's Coloured Progressive Matrices & $19 \cdot 8(5 \cdot 5)$ & $22 \cdot 1(6 \cdot 7)$ & $2.33(0.28$ to 4.38$)$ & 0.026 & $1.29(-1.36$ to 3.93$)$ & $0 \cdot 34$ \\
\hline Digit Forwards & $6 \cdot 8(2 \cdot 1)$ & $7 \cdot 3(2 \cdot 5)$ & $0.51(-0.26$ to $1 \cdot 27)$ & $0 \cdot 19$ & $0.57(-0.43$ to 1.56$)$ & $0 \cdot 26$ \\
\hline Digit Backwards & $3 \cdot 2(1 \cdot 6)$ & $3 \cdot 6(2 \cdot 1)$ & $0.47(-0 \cdot 16$ to $1 \cdot 1)$ & $0 \cdot 15$ & $0.46(-0.34$ to $1 \cdot 26)$ & $0 \cdot 25$ \\
\hline
\end{tabular}

Data are mean (SD) unless otherwise stated. Data were analysed using ANCOVA, with age, sex, parental loss, HIV disclosure, previous treatment failure, combination antiretroviral therapy duration, history of tuberculosis treatment, and previous hospital admission entered as covariates in the adjusted models.

* One child in the efavirenz group was excluded from the Child Behavior Checklist competence analyses because of missing data. 
Table 3. Central nervous system symptoms

\begin{tabular}{|c|c|c|c|c|c|c|}
\hline & \multirow{2}{*}{$\begin{array}{l}\text { Non-efavirenz } \\
(n=69)\end{array}$} & \multirow{2}{*}{$\begin{array}{c}\text { Efavirenz } \\
\quad(n=72)\end{array}$} & \multirow{2}{*}{$\begin{array}{c}\text { Crude model } \\
\text { Odds ratio }(95 \% \mathrm{CI})\end{array}$} & \multicolumn{3}{|c|}{ Adjusted model } \\
\hline & & & & P-value & Odds ratio $(95 \% \mathrm{CI})$ & P-value \\
\hline Abnormal dreaming & $44(64 \%)$ & $35(49 \%)$ & $0.54(0.27$ to 1.05$)$ & $0 \cdot 071$ & $0.32(0.11$ to 0.9$)$ & $0 \cdot 031$ \\
\hline Difficulty going to sleep & $9(13 \%)$ & $5(7 \%)$ & $0.50(0.16$ to 1.57$)$ & $0 \cdot 23$ & $0.29(0.06$ to 1.55$)$ & $0 \cdot 15$ \\
\hline Difficulty staying asleep & $21(30 \%)$ & $14(19 \%)$ & $0.55(0 \cdot 25$ to $1 \cdot 2)$ & $0 \cdot 13$ & $0 \cdot 35(0 \cdot 11$ to $1 \cdot 15)$ & $0 \cdot 083$ \\
\hline $\begin{array}{l}\text { Dizziness or balance } \\
\text { problems }\end{array}$ & $15(22 \%)$ & $14(19 \%)$ & $0.87(0.38$ to 1.97$)$ & $0 \cdot 74$ & $1 \cdot 01(0 \cdot 31$ to $3 \cdot 35)$ & 0.98 \\
\hline Feelings of uneasiness & $12(17 \%)$ & $20(28 \%)$ & $1.83(0.81$ to $4 \cdot 1)$ & $0 \cdot 14$ & $3.45(1.02$ to 11.63$)$ & $0 \cdot 046$ \\
\hline Lack of vivacity ${ }^{*}$ & $3(4 \%)$ & $5(7 \%)$ & $1.64(0 \cdot 38$ to $7 \cdot 15)$ & $0 \cdot 51$ &.. &.. \\
\hline Depressed or anxious $^{\dagger}$ & $6(9 \%)$ & $17(24 \%)$ & $3 \cdot 25(1.20$ to $8 \cdot 81)$ & $0 \cdot 021$ & .. &.. \\
\hline Attention problems at school & $8(12 \%)$ & $19(26 \%)$ & $2.73(1.11$ to 6.75$)$ & $0 \cdot 029$ & $6.92(1.81$ to $26 \cdot 48)$ & $0 \cdot 0047$ \\
\hline
\end{tabular}

Data are $n(\%)$ unless otherwise stated. Presence (yes or no) of symptoms in the week before the study visit (including the day of study visit) was reported by the child and caretaker. Data were analysed with multiple logistic regression analysis, with age, sex, parental loss, HIV disclosure, previous treatment failure, combination antiretroviral therapy duration, history of tuberculosis treatment, and previous hospital admission entered as covariates in the adjusted models.

* Given the small number of events, the adjusted model was not applied.

+ Given the substantial impact of the covariate previous treatment failure on the adjusted model, together with the low occurrence of treatment failure in the efavirenz group, no adjusted model was applied. 
When comparing the prescribed efavirenz dose with the dose recommended in the WHO 2016 guidelines, ${ }^{3}$ we observed that $40(56 \%)$ of 71 children in the efavirenz group had been prescribed the recommended dose, with $28(39 \%)$ of 71 children receiving lower doses and three $(4 \%)$ of 71 children receiving higher doses than recommended (figure). Children in the low-dose efavirenz group did not differ from children in the recommended and high-dose efavirenz group in age $(\mathrm{p}=0.69)$, sex $(\mathrm{p}=0.22)$, or number of children with less than 40 copies of HIV RNA per mL ( $\mathrm{p}=1 \cdot 00$; appendix $\mathrm{p} 10)$. To assess whether under-dosing of efavirenz had affected our results, we repeated our analyses after excluding children who were prescribed lower doses than recommended by WHO (appendix p 11). In line with our initial analyses, we observed lower competence scores in children in the efavirenz group $(n=43)$ than in those in the non-efavirenz group ( $n=69$; adjusted mean difference $-2 \cdot 55[95 \% \mathrm{CI}-4 \cdot 56$ to $-0 \cdot 55], \mathrm{p}=0 \cdot 013$ ) as well as lower school performance scores (adjusted mean difference $-1.06[-1.61$ to -0.50$], \mathrm{p}=0.00027$; appendix $\mathrm{p} 11)$. In addition, children in the efavirenz group had higher total problem scores (adjusted mean difference 8.92 [95\% CI 1.24-16.60], p=0.023) and internalising problem scores (adjusted mean difference 3.11 [0.60-5.63], $\mathrm{p}=0 \cdot 016$ ), but no difference in externalising problem scores (adjusted mean difference $0 \cdot 82$ [-1.70 to $3 \cdot 34], \mathrm{p}=0 \cdot 52$ ) compared with children in the nonefavirenz group (appendix p 11). Post-hoc analyses of the individual internalising syndromes showed that children in the efavirenz group had higher scores on anxious and depressed behaviours (adjusted mean difference $0 \cdot 84$ [95\% CI $-0 \cdot 12$ to $1 \cdot 80], \mathrm{p}=0 \cdot 087$ ), withdrawn behaviour (adjusted mean difference $1 \cdot 17$ [ $-0 \cdot 11$ to $2 \cdot 44$ ], $\mathrm{p}=0 \cdot 073$ ), and somatic complaints (adjusted mean difference $1 \cdot 11[-0 \cdot 03$ to $2 \cdot 25], \mathrm{p}=0 \cdot 057$ ) than children in the non-efavirenz group (appendix p 11), but these results were not significant. Finally, children in the efavirenz group scored higher for thought problems (adjusted mean difference 1.25 [95\% CI 0.16-2.34], p=0.025) and attention problems (adjusted mean difference $1 \cdot 60$ [0.03-3.17], p=0.046) than did children in the non-efavirenz group (appendix p 11). Results for non-adherence (appendix p 9) and cognitive test performance (appendix p 11) did not change. Further exclusion of three children who were prescribed higher doses than recommended did not substantially alter these findings (appendix p 12) 


\section{Discussion}

n this cross-sectional, observational study, we found that efavirenz-based cART was associated with lower total competence and school performance scores among long-term treated Tanzanian children with HIV infection than was non-efavirenz-based cART. Higher total problem and internalising problem scores were also observed in children in the efavirenz group than in those in the non-efavirenz group, although these results were not significant. We found no difference in cognitive performance and adherence between children on efavirenz and children on alternative cART. Except for externalising problems, the effects on problem scores became more pronounced after exclusion of children who received low efavirenz doses.

The most striking findings in our study were the lower competence and school performance scores in children on efavirenz than in children on alternative cART. Since groups were equally intelligent (RCPM), and short-term and working memory seemed unaffected (digit span), these factors cannot explain the differences in school performance. Hence, other factors, such as other neurocognitive problems or mood problems, might have played a part. For example, $19(26 \%)$ of 72 children on efavirenz reported problems paying attention at school compared with eight (12\%) of 69 children in the non-efavirenz group (table 3). Follow-up research into these attentional control networks (inhibiting irrelevant distractions, sustaining attention, dividing attention, and shifting attention) might be fruitful.

Second, our results suggest that children on efavirenz have increased psychopathology. We observed higher total and internalising problems in children on efavirenz than in children on alternative cART, with internalising problems being characterised by anxious and affective symptoms. The effects were more pronounced in an added subgroup analysis among children who were prescribed doses higher than or equal to WHO recommended doses. In addition, children on efavirenz more frequently reported symptoms of depression or anxiety and feelings of uneasiness (table 3). Our results differ from findings by Coovadia and colleagues,

12, 13 who reported no difference in psychopathology, defined by the SDQ, between South African children on efavirenz and children on ritonavir-boosted lopinavir. These differences could be partly explained by differences in age (with mean age 4.3 years in their population vs 9.6 years in our population), culture, and ethnicity. In addition, different psychiatric tools were used to define psychopathology. Both the SDQ and the CBCL are considered to be effective screeners for identification of child psychopathology. ${ }^{24}$ The SDQ is shorter, does not have a section on school performance, and has slightly lower sensitivity compared with the CBCL. ${ }^{24}$ Subtle, internalising symptoms might have been overlooked with the SDQ. ${ }^{13}$ In adults on long-term efavirenz, internalising problems, such as symptoms of depression, anxiety, and stress, also dominate the neuropsychiatric side-effect profile. ${ }^{6,25}$ Overall, these symptoms seem to be more pronounced in adults than in children, with greater consequences for quality of life and treatment discontinuation. ${ }^{11}$ It is possible that children, because of differential predisposition to drug side-effects, simply suffer less from CNS side-effects of efavirenz than do adults. However, caretakers generally consider internalising problems less problematic because these problems are characterised by internal distress, rather than overtly socially negative or disruptive behaviour. These features make internalising problems difficult to detect, especially when relying on parent-based or caretaker-based reports, as is the case with our study. Finally, children on efavirenz less frequently reported signs of abnormal 
dreaming than did children on alternative cART (table 3), probably because all children from our study received efavirenz for 6 months or more, and this and similar problems are thought to resolve after the first weeks of treatment. ${ }^{13}$ Importantly, the course of psychiatric symptoms in children is hard to predict, especially during crucial periods such as transition to adolescence and adulthood. For example, our group ${ }^{25}$ recently showed that depression scores were reduced in adolescents, but increased in adults, on efavirenz in Tanzania. The increasing evidence for higher risks of suicidality among adults on efavirenz than among adults on non-efavirenz-based cART emphasises the need for clinical follow-up of neuropsychiatric symptoms in children on efavirenz. ${ }^{26}$ Finally, in-vitro and animal studies have shown that efavirenz can interfere with cell energy homoeostasis and interact with the brain serotonin system. ${ }^{27}$ Serotonin fulfils an important role for brain development, and exposure to serotonergic drugs (eg, antidepressants of the class serotonin reuptake inhibitors) during childhood or pregnancy has been linked to behavioural and developmental problems. ${ }^{28}$ Future studies are needed to assess long-term developmental consequences of paediatric efavirenz.

Exclusion of children on suboptimal efavirenz doses increased group differences in behavioural and emotional problems. No specific indications for efavirenz dose reduction were recorded in the clinical files of these children. Moreover, we did not observe differences in general characteristics or viral suppression between children in the low-dose efavirenz group and the recommended and high-dose efavirenz group. Efavirenz has a narrow therapeutic window, and a plasma concentration of less than $1000 \mathrm{ng} / \mathrm{mL}$ has been consistently linked to virological failure and a plasma concentration of more than $4000 \mathrm{ng} / \mathrm{mL}$ has been linked to CNS toxicity. ${ }^{29}$ In addition to drug dose, efavirenz exposure is determined by adherence, absorption, and metabolism. ${ }^{7}$ Efavirenz is metabolised in the liver by cytochrome P450-2B6 (CYP2B6), and certain genetic variants of this enzyme have been strongly associated with higher plasma concentrations. These genetic variants are more common in subSaharan Africa (eg, the $516 \mathrm{G} \rightarrow \mathrm{T}$ substitution is prevalent in $48 \cdot 8 \%$ of African ancestry). ${ }^{30}$ We did not measure plasma concentrations of efavirenz in our study, and the results from our subgroup analysis have to be interpreted with caution. Nevertheless, they might also support dose dependency of efavirenz-related neuropsychiatric symptoms.

The major strength of our study is that, by applying standardised validated paediatric psychiatric tools, we were able to comprehensively assess emotions, behaviours, and cognition in a substantially large, representative sample of school-aged children with HIV infection in sub-Saharan Africa. Different limitations should also be acknowledged. First, we did not measure efavirenz plasma concentrations or CD4 cell counts. Second, we cannot completely rule out effects of HIV on the brain in view of the finding that 33 (23\%) of 141 children had HIV RNA counts of 40-1000 copies per mL (table 1) and data about cumulative exposure and HIV RNA counts in the cerebrospinal fluid were unavailable. Third, no blinding was applied. Nevertheless, we consider it unlikely that the lack of blinding had an important confounding effect because assessments were highly standardised and followed the protocol strictly, and study nurses recorded scores without interpreting behaviour. Finally, the cross-sectional observational design of our study comes with limitations, and no causal relationships can be identified. Groups differed for several clinical parameters, parental loss, and disclosure of HIV. Overall, the nonefavirenz group had a more severe clinical course, probably leading to underestimation of the effects of efavirenz 
in the crude models. Although we tried to correct for these clinical factors by adding them to our statistical models, we cannot rule out underestimation of the severity of the side-effect profile of efavirenz.

In conclusion, use of efavirenz in our paediatric cohort was associated with a mild increase in neuropsychiatric symptoms, especially in children who received doses higher than or equal to the WHO recommended doses for efavirenz. These findings, together with the pervasive evidence of efavirenz-related side-effects in adults and serotonergic properties of efavirenz, highlight the need for roll-out of paediatric formulations of alternative antiretroviral drugs. Until then, clinical awareness of neuropsychological symptoms remains warranted for children on efavirenz. This clinical awareness requires development of culturally adapted instruments and training of more mental health workers, especially in low-income settings, such as Tanzania. If symptoms are present in efavirenz-treated children, close follow-up is indicated. Referral for psychosocial support or switching to alternative cART should be considered if symptoms persist. 


\section{Contributors}

LVdW, DNM, QdM, BTM, NNJR, AD, AJAMvdV, AFAS, and GDK designed the study. LVdW, DNM, and AD drafted the study protocol. LVdW, DNM, BTM, and GDK coordinated the study inclusion. LVdW, NNJR, and AFAS analysed the data. LVdW, DNM, QdM, NNJR, AJAMvdV, AFAS, and GDK interpreted the data. LVdW wrote the manuscript. All authors critically reviewed and revised the manuscript. All authors approved the final manuscript.

\section{Conflict of interest}

We declare no competing interests.

\section{Acknowledgements}

This work was supported by Aidsfonds (P-18802) and Radboud University Medical Center (51523). We thank all children and their caretakers for their participation in the study. We thank the local staff of the participating institutions for their commitment and support with participant recruitment. We thank Zawadiel Hillu, Stella Mbiri, Neema Mahimbo, Yukunda Mrema, Adeline Lyimo, and Rose Mosile for their extensive work on the interviews and assessments. We thank Kilimanjaro Christian Medical Centre and Kilimanjaro Clinical Research Institute for administrative support during the study. We thank Rogier Donders for his help with the statistical analyses. Finally, we acknowledge the regional medical officer, Best Magoma, and the district medical officers of Moshi Urban and Moshi Rural for their permission and help with this study. 


\section{References}

1 UNAIDS. Global HIV \& AIDS statistics-2018 fact $\quad$ sheet. 2018. http://www.unaids.org/sites/default/files/media_asset/UNAIDS_FactSheet_en.pdf (accessed Jan 31, 2019).

${ }^{2}$ Ministry of Health and Social Welfare, Tanzania. National Guidelines for the Management of HIV and AIDS, 5th edn. Dar es Salaam: National AIDS Control Programme, 2015.

${ }^{3}$ WHO. Consolidated guidelines on the use of antiretroviral drugs for treating and preventing HIV infection. Recommendations for a public health approach, 2nd edn. Geneva: World Health Organization, 2016.

${ }^{4}$ Murnane PM, Strehlau R, Shiau S, et al. Switching to efavirenz versus remaining on ritonavir-boosted lopinavir in HIV-infected children exposed to nevirapine: long-term outcomes of a randomized trial. Clin Infect Dis 2017; 65: 477-85.

${ }^{5}$ Ford N, Shubber Z, Pozniak A, et al. Comparative safety and neuropsychiatric adverse events associated with efavirenz use in first-line antiretroviral therapy: a systematic review and meta-analysis of randomized trials. $J$ Acquir Immune Defic Syndr 2015; 69: 422-29.

6 Mothapo KM, Schellekens A, Van Crevel R, et al. Improvement of depression and anxiety after discontinuation of long-term efavirenz treatment. CNS Neurol Disord Drug Targets 2015; 14: 811-18.

${ }^{7}$ Apostolova N, Funes HA, Blas-Garcia A, Galindo MJ, Alvarez A, Esplugues JV. Efavirenz and the CNS: what we already know and questions that need to be answered. J Antimicrob Chemother 2015;70: 2693-708.

${ }^{8}$ Ciccarelli N, Fabbiani M, Di Giambenedetto S, et al. Efavirenz associated with cognitive disorders in otherwise asymptomatic HIV-infected patients. Neurology 2011; 76: 1403-09.

9 WHO. Updated recommendations on first-line and second-line antiretroviral regimes and post-exposure prophylaxis and recommendations on early infant diagnosis of HIV. Geneva: World Health Organization, 2018: 13.

${ }^{10}$ Starr SE, Fletcher CV, Spector SA, et al. Combination therapy with efavirenz, nelfinavir, and nucleoside reverse-transcriptase inhibitors in children infected with human immunodeficiency virus type 1. Pediatric AIDS Clinical Trials Group 382 Team. N Engl J Med 1999; 341: 1874-81.

${ }^{11}$ Van de Wijer L, Schellekens AFA, Burger DM, Homberg JR, de Mast Q, van der Ven A. Rethinking the riskbenefit ratio of efavirenz in HIV-infected children. Lancet Infect Dis 2016; 16: e76-81.

${ }^{12}$ Coovadia A, Abrams EJ, Strehlau R, et al. Efavirenz-based antiretroviral therapy among nevirapine-exposed HIV-infected children in South Africa: a randomized clinical trial. JAMA 2015; 314: 1808-17.

${ }^{13}$ Murnane PM, Strehlau R, Shiau S, et al. Reply to Van de Wijer et al. Clin Infect Dis 2018; 66: 1151-52.

${ }^{14}$ Teglas JP, Quartier P, Treluyer JM, Burgard M, Gregoire V, Blanche S. Tolerance of efavirenz in children. AIDS 2001; 15: 241-43.

${ }^{15}$ Soeria-Atmadja S, Osterberg E, Gustafsson LL, et al. Genetic variants in CYP2B6 and CYP2A6 explain interindividual variation in efavirenz plasma concentrations of HIV-infected children with diverse ethnic origin. PLoS One 2017; 12: e0181316.

${ }^{16}$ Wynberg E, Williams E, Tudor-Williams G, Lyall H, Foster C. Discontinuation of efavirenz in paediatric patients: why do children switch? Clin Drug Invest 2017; 38: 231-38. 
${ }^{17}$ Pinillos F, Dandara C, Swart M, et al. Case report: severe central nervous system manifestations associated with aberrant efavirenz metabolism in children: the role of CYP2B6 genetic variation. BMC Infect Dis 2016; 16: 56.

18 Achenbach TM, Rescorla LA. Manual for the ASEBA school-age forms and profiles. Burlington, VT: ASEBA, 2001.

${ }^{19}$ Louw KA, Ipser J, Phillips N, Hoare J. Correlates of emotional and behavioural problems in children with perinatally acquired HIV in Cape Town, South Africa. AIDS Care 2016; 28: 842-50.

${ }^{20}$ Costenbader V, Ngari SM. A Kenya standardization of the Raven's Coloured Progressive Matrices. School Psychol Int 2001; 22: 258-68.

${ }^{21}$ Wechsler D. Wechsler Intelligence Scale for Children, 5th edn. Boston, MA: Pearson, 2014.

${ }^{22}$ Sherr L, Hensels IS, Tomlinson M, Skeen S, Macedo A. Cognitive and physical development in HIV-positive children in South Africa and Malawi: a community-based follow-up comparison study. Child Care Health Dev 2018; 44: 89-98.

${ }^{23}$ Harrison L, Ananworanich J, Hamadache D, et al. Adherence to antiretroviral therapy and acceptability of planned treatment interruptions in HIV-infected children. AIDS Behav 2013; 17: 193-202.

${ }^{24}$ Warnick EM, Bracken MB, Kasl S. Screening efficiency of the child behavior checklist and strengths and difficulties questionnaire: a systematic review. Child Adolesc Ment Health 2008; 13: 140-47.

${ }^{25}$ Sumari-de Boer M, Schellekens A, Duinmaijer A, et al. Efavirenz is related to neuropsychiatric symptoms among adults, but not among adolescents living with human immunodeficiency virus in Kilimanjaro, Tanzania. Trop Med Int Health 2017; 23: 164-72.

${ }^{26}$ Arenas-Pinto A, Grund B, Sharma S, et al. Risk of suicidal behavior with use of efavirenz: results from the strategic timing of antiretroviral treatment trial. Clin Infect Dis 2018; 67: 420-29.

${ }^{27}$ Dalwadi DA, Kim S, Amdani SM, Chen Z, Huang RQ, Schetz JA. Molecular mechanisms of serotonergic action of the HIV-1 antiretroviral efavirenz. Pharmacol Res 2016; 110: 10-24.

${ }^{28}$ Homberg JR, Schubert D, Gaspar P. New perspectives on the neurodevelopmental effects of SSRIs. Trends Pharmacol Sci 2010;31: 60-65.

${ }^{29}$ Marzolini C, Telenti A, Decosterd LA, Greub G, Biollaz J, Buclin T. Efavirenz plasma levels can predict treatment failure and central nervous system side effects in HIV-1-infected patients. AIDS 2001; 15: 71-75.

${ }^{30}$ Klein K, Lang T, Saussele T, et al. Genetic variability of CYP2B6 in populations of African and Asian origin: allele frequencies, novel functional variants, and possible implications for anti-HIV therapy with efavirenz. Pharmacogenet Genomics 2005; 15: 861-73. 


\section{Research in context panel}

Evidence before this study

Neuropsychiatric side-effects of efavirenz in adults with HIV infection are increasingly being acknowledged. Whether neuropsychiatric side-effects of efavirenz are also common in children remains unclear. To identify paediatric studies on neuropsychiatric side-effects of efavirenz, we searched PubMed on June 6, 2018, using the search terms "efavirenz" AND "children", including relevant synonyms (see appendix p 2 for the extensive search strategy). The search was unrestricted by language or publication date and yielded 987 search results. After manual title and abstract screening, we selected 158 paediatric studies evaluating clinical outcomes of efavirenz. Full-text screening of these papers and relevant references resulted in 39 relevant papers. Two studies used paediatric psychiatric assessment tools. One trial compared psychopathology between children receiving ritonavir-boosted lopinavir versus efavirenz and found no differences in emotional problem scores between groups using the 25-item Strengths and Difficulties Questionnaire (SDQ). Another observational study reported better SDQ scores in adolescents on efavirenz than in those on non-efavirenz-containing regimens. By contrast, five case reports described severe neuropsychiatric symptoms, including ataxia, psychotic symptoms, and seizures, during the course of efavirenz treatment, which were linked to high efavirenz plasma concentrations in three papers. The remaining 32 papers reported clinical observations of neuropsychiatric symptoms in trials and observational studies, without using psychiatric tools. These studies were largely inconclusive, with some showing prevalence of up to $39 \%$.

\section{Added value of this study}

Using validated psychiatric tools, we present the first comprehensive psychiatric evaluation of children with HIV infection in sub-Saharan Africa on efavirenz. Our findings show an association between paediatric efavirenz and a mild increase in neuropsychiatric symptoms, especially in children on adequate or above recommended doses by WHO.

\section{Implications of all the available evidence}

WHO recommends efavirenz as a key component of paediatric HIV treatment. Our findings emphasise the need for further paediatric behavioural studies comparing efavirenz with alternative, newer drugs. Meanwhile, early detection and adequate follow-up of neuropsychological symptoms inefavirenz-treated children should be made a priority. 